\title{
Características clínicas de los pacientes con fiebre manchada de las Montañas Rocosas, infección por dengue y chikungunya
}

\author{
Jesús Delgado-de la Mora, ${ }^{1}$ Jesús D. Licona-Enríquez, ${ }^{2}$ Diego I. Álvarez-López, ${ }^{3}$ Diana E. Aguilar-León ${ }^{1}$ \\ y Gerardo Álvarez-Hernández ${ }^{3 *}$ \\ ${ }^{1}$ Instituto Nacional de Ciencias Médicas y Nutrición "Salvador Zubirán", Departamento de Anatomía Patológica, Ciudad de México; ${ }^{2}$ nstituto \\ Mexicano del Seguro Social, Centro Médico Nacional Siglo XXI, Hospital de Pediatría "Silvestre Frenk Freund", Ciudad de México; ${ }^{3 U n i v e r s i d a d ~}$ \\ de Sonora, Departamento de Medicina y Ciencias de la Salud, Sonora. México
}

\section{Resumen}

Introducción: La distinción clínica entre infecciones arbovirales y las provocadas por rickettsias es crucial para iniciar el tratamiento médico apropiado. Objetivo: Comparar las diferencias entre fiebre manchada de las Montañas Rocosas (FMMR) y otras enfermedades transmitidas por vector (dengue y chikungunya) con presentación clínica similar e identificar los datos que pudieran ayudar al diagnóstico rápido de esas enfermedades. Métodos: Se evaluaron datos sociodemográficos, clínicos y de laboratorio de 399 pacientes de cinco hospitales y clínicas en Sonora, México, entre 2004 y 2016, con el diagnóstico confirmado por laboratorio de FMMR, dengue o chikungunya. Resultados: El grupo con FMMR presentó la mayor letalidad (49/63 muertes, $77.8 \%)$, seguido por el de chikungunya (3/161, 1.9\%) y el de dengue (3/161, 1.9\%). Las diferencias clínicas consistieron en la presencia de exantema, edema y prurito; además, se documentaron diferencias en múltiples biomarcadores como plaquetas, hemoglobina, bilirrubina indirecta y niveles de sodio sérico. Conclusión: El exantema en palmas y plantas, edema y ausencia de prurito, aunados a niveles altos de bilirrubina directa y trombocitopenia severa pudieran ser indicadores útiles para diferenciar a pacientes con FMMR en etapas avanzadas de aquellos con dengue y chikungunya.

PALABRAS CLAVE: Fiebre manchada de las Montañas Rocosas. Dengue. Chikungunya. México.

\section{Clinical features of patients with Rocky Mountain spotted fever, dengue and chikungunya infection}

\begin{abstract}
Introduction: Clinical distinction between arbovirus infections and those caused by rickettsia is crucial to initiate appropriate medical treatment. Objective: To compare the differences between Rocky Mountain spotted fever (RMSF) and other vector-borne diseases (dengue and chikungunya) with similar clinical presentation, and to identify data that could aid rapid diagnosis of these diseases. Methods: Sociodemographic, clinical and laboratory data of 399 patients from five hospitals and clinics of Sonora, Mexico, with laboratory-confirmed diagnosis of RMSF, dengue, or chikungunya between 2004 and 2016 were evaluated. Results: The RMSF group had the highest lethality (49/63 deaths, $77.8 \%)$, followed by the chikungunya group (3/161, 1.9\%) and the dengue group $(3 / 161,1.9 \%)$. Clinical differences included the presence of rash, edema, and pruritus; in addition, differences in multiple biomarkers such as platelets, hemoglobin, indirect bilirubin, and serum sodium levels were documented. Conclusion: Rash on the palms and soles, edema and absence of pruritus, together with high levels of direct bilirubin and severe thrombocytopenia could be useful indicators to differentiate patients at RMSF advanced stages from those with dengue and chikungunya.
\end{abstract}

KEY WORDS: Rocky Mountain spotted fever. Dengue. Chikungunya. Mexico.

Correspondencia:

*Gerardo Álvarez-Hernández

E-mail: galvarezh63@gmail.com

0016-3813/@ 2020 Academia Nacional de Medicina de México, A.C. Publicado por Permanyer. Este es un artículo open access bajo la licencia CC BY-NC-ND (http://creativecommons.org/licenses/by-nc-nd/4.0/).
Fecha de recepción: 07-03-2020

Fecha de aceptación: 07-07-2020

DOI: $10.24875 / G M M .20000105$
Gac Med Mex. 2021;157:61-66

Disponible en PubMed

www.gacetamedicademexico.com 


\section{Introducción}

La presentación clínica de muchas enfermedades transmitidas por vector, incluida la mayoría de las infecciones arbovirales y las originadas por rickettsias, representa un reto para los clínicos, particularmente debido a la inespecificidad de los signos y síntomas tempranos al comienzo de las enfermedades, cuando es necesario un diagnóstico de trabajo para iniciar apropiadamente el tratamiento médico. Actualmente, el estado de Sonora, localizado en el noroeste de México, es una región donde la fiebre manchada de las Montañas Rocosas (FMMR) enfermedad bacteriana transmitida por garrapatas-, el dengue y la chikungunya -infecciones arbovirales transmitidas por mosquitos- son endémicos y frecuentemente se presentan en forma simultánea.

La FMMR, causada por Rickettsia rickettsii, está asociada a altas tasas de letalidad, que varían entre 28 y $40 \%$ en pacientes sin tratamiento. ${ }^{1-3}$ Los virus de dengue y chikungunya son trasmitidos por mosquitos del género Aedes y las infecciones que ocasionan están característicamente asociadas a bajas tasas de letalidad. ${ }^{4,5}$

La distinción clínica entre estas enfermedades es crucial. El manejo inicial del dengue y la chikungunya es principalmente sintomático y en los casos graves se limita a medidas de soporte..$^{4,5} \mathrm{El}$ inicio rápido de la terapia con doxiciclina es crucial para reducir el riesgo de muerte en pacientes con FMMR. ${ }^{1}$ No obstante, ningún estudio ha evaluado específicamente las diferencias clínicas y de laboratorio entre pacientes con tales enfermedades. Aquí describimos y comparamos una serie de casos de pacientes que viven en Sonora diagnosticados con FMMR, dengue 0 chikungunya.

\section{Métodos}

Estudio transversal de una serie de 399 pacientes con FMMR, dengue o chikungunya confirmados por laboratorio, que recibieron atención en cinco establecimientos de salud pública del estado de Sonora, hospitales y clínicas periféricas, entre el 1 de enero de 2004 y el 31 de diciembre de 2016. Un caso de FMMR fue un sujeto con una prueba positiva de reacción en cadena de polimerasa (PCR) para Rickettsia rickettsii, en la que se utilizaron los primers CS-78 y CD-323 para amplificar un fragmento de $401 \mathrm{pb}$ del gen gltA de especies de Rickettsia, así como los primers RRI1 y RRI2 para la proteína hipotética A1G_04230 de Rickettsia rickettsii; ${ }^{6,7}$ o una cuadruplicación de títulos entre muestras pareadas de suero por ensayo de anticuerpos de inmunofluorescencia indirecta para Rickettsia rickettsii. Se consideró caso de dengue o chikungunya al individuo con un resultado positivo para la prueba del antígeno NS1 (solo en dengue) o una prueba de PCR positiva en una muestra de suero. La información clínica fue recolectada por los médicos de los expedientes clínicos, formas epidemiológicas y certificados de defunción. Antes de la recolección de los datos, el comité de ética de la Universidad de Sonora y de cada uno de los hospitales participantes aprobaron el protocolo de investigación, que quedó registrado como 13CEI26030258, 13CEI26030259, 13CEI26030260.

Se empleó estadística descriptiva para caracterizar el perfil clínico y de laboratorio de los sujetos de estudio. Para evaluar la diferencia entre los sujetos de estudio, en las variables categóricas se utilizó prueba de chi cuadrada y la normalidad de los datos de las variables cualitativas fue evaluada con prueba de Shapiro-Wilk. La comparación fue realizada con ANOVA si se trató de distribución normal o prueba de Kruskal-Wallis si no lo fue. Consideramos los resultados con un valor de $p<0.05$ como estadísticamente significativos. Los datos fueron analizados mediante el programa estadístico Number Crunching Statistical System versión 9.0.

\section{Resultados}

Durante el periodo de 13 años de evaluación hubo 63 casos (15.8\%) con diagnóstico de FMMR, incluidos 49 que fallecieron ( $77.8 \%$ ); 175 casos de dengue $(43.9 \%)$, con tres defunciones (1.7\%); y 161 casos $(40.3 \%)$ de chikungunya, con dos defunciones $(1.24 \%)$. Del grupo con FMMR, la mayoría se trató de hombres (61.9\%), lo cual difirió significativamente $(p<0.0 .1)$ del grupo con dengue $(43.7 \%)$ y chikungunya $(41.1 \%)$. Los 63 pacientes del grupo con FMMR fueron hospitalizados (100\%), mientras que 50 pacientes con dengue $(28.6 \%)$ y ocho con chikungunya $(5.0 \%)$ requirieron hospitalización. El promedio de días de evolución al momento de la primera evaluación médica fue mayor en el grupo con FMMR (5.5 \pm 2.4$)$, que en los pacientes con dengue $(3.0 \pm 1.9)$ y chikungunya $(4.4 \pm 2.9), p<0.01$ (Tabla 1).

La fiebre fue el hallazgo inicial predominante $(83.1 \%)$ en los pacientes con dengue, el exantema 
Tabla 1. Características seleccionadas al momento de la admisión hospitalaria en 399 pacientes con fiebre manchada de las Montañas Rocosas, dengue y chikungunya. Sonora, Mexico. 2004-2016

\begin{tabular}{|c|c|c|c|c|c|c|c|c|c|c|}
\hline \multirow{2}{*}{$\begin{array}{l}\text { Caracteristica } \\
\text { Letalidad }\end{array}$} & \multicolumn{2}{|c|}{$\begin{array}{c}\text { FMMR }<5 \text { días de } \\
\text { evolución } \\
(n=18)\end{array}$} & \multicolumn{2}{|c|}{$\begin{array}{l}\text { FMMR } \geq 5 \text { días de } \\
\text { evolución } \\
(n=45)\end{array}$} & \multicolumn{2}{|c|}{$\begin{array}{l}\text { Dengue } \\
(n=175)\end{array}$} & \multicolumn{2}{|c|}{$\begin{array}{l}\text { Chikungunya } \\
\qquad(\mathrm{n}=161)\end{array}$} & \multirow{2}{*}{$\begin{array}{l}p_{1}^{*} \\
0.73\end{array}$} & \multirow{2}{*}{$\begin{array}{l}p_{2}^{* *} \\
<0.01\end{array}$} \\
\hline & 13 & 72.2 & 36 & 80.0 & 3 & 1.7 & 3 & 1.9 & & \\
\hline Hospitalización & 18 & 100.0 & 45 & 100.0 & 50 & 28.6 & 8 & 5.0 & 1.00 & $<0.01^{\dagger}$ \\
\hline Sexo (hombre) & 11 & 61.1 & 28 & 62.2 & 67 & 38.3 & 66 & 41.0 & 0.93 & $0.01^{\dagger}$ \\
\hline Fiebre & 11 & 61.1 & 33 & 73.3 & 143 & 81.7 & 65 & 40.4 & 0.51 & $<0.01^{\dagger}$ \\
\hline $\begin{array}{l}\text { Exantema } \\
\text { Plantas } \\
\text { Palmas } \\
\text { Palmas y plantas }\end{array}$ & $\begin{array}{l}18 \\
17 \\
17 \\
15\end{array}$ & $\begin{array}{l}100.0 \\
94.4 \\
94.4 \\
83.3\end{array}$ & $\begin{array}{l}43 \\
40 \\
41 \\
40\end{array}$ & $\begin{array}{l}95.6 \\
88.9 \\
91.1 \\
88.9\end{array}$ & $\begin{array}{c}59 \\
2 \\
3 \\
2\end{array}$ & $\begin{array}{c}33.7 \\
1.1 \\
1.7 \\
1.1\end{array}$ & $\begin{array}{l}77 \\
16 \\
16 \\
14\end{array}$ & $\begin{array}{c}47.8 \\
9.9 \\
9.9 \\
8.9\end{array}$ & $\begin{array}{l}0.91 \\
0.84 \\
0.66 \\
0.86\end{array}$ & $\begin{array}{l}<0.01^{\dagger} \\
<0.01^{\dagger} \\
<0.01^{\dagger} \\
<0.01^{\dagger}\end{array}$ \\
\hline $\begin{array}{l}\text { Edema } \\
\text { Tobillo } \\
\text { Muñeca } \\
\text { Párpados }\end{array}$ & $\begin{array}{l}3 \\
5 \\
6\end{array}$ & $\begin{array}{l}16.7 \\
27.8 \\
33.3\end{array}$ & $\begin{array}{l}15 \\
14 \\
14\end{array}$ & $\begin{array}{l}33.3 \\
31.1 \\
31.1\end{array}$ & $\begin{array}{l}4 \\
3 \\
0\end{array}$ & $\begin{array}{l}2.3 \\
1.7 \\
0.0\end{array}$ & $\begin{array}{c}10 \\
6 \\
2\end{array}$ & $\begin{array}{l}6.2 \\
3.7 \\
1.2\end{array}$ & $\begin{array}{l}0.31 \\
0.79 \\
0.86\end{array}$ & $\begin{array}{l}<0.01^{\dagger} \\
<0.01^{\dagger} \\
<0.01^{\dagger}\end{array}$ \\
\hline Prurito & 0 & 0.0 & 0 & 0.0 & 23 & 13.1 & 37 & 23.0 & 1.00 & $<0.01^{\dagger}$ \\
\hline Hepatomegalia & 7 & 38.9 & 7 & 15.6 & 0 & 0.0 & 0 & 0.0 & 0.10 & $<0.01^{\dagger}$ \\
\hline Vómito & 8 & 44.4 & 18 & 40.0 & 19 & 10.9 & 11 & 6.9 & 0.97 & $<0.01^{+}$ \\
\hline Cefalea & 11 & 61.1 & 29 & 64.4 & 123 & 70.3 & 89 & 55.3 & 0.80 & $0.04^{\dagger}$ \\
\hline Mialgias & 12 & 66.7 & 32 & 71.1 & 118 & 67.4 & 111 & 68.9 & 0.97 & 0.96 \\
\hline Artralgias & 12 & 66.7 & 29 & 64.4 & 118 & 67.4 & 142 & 88.2 & 0.87 & $<0.01^{\dagger}$ \\
\hline Hemorragia & 1 & 5.6 & 5 & 11.1 & 14 & 8.0 & 4 & 2.5 & 0.84 & 0.07 \\
\hline Dolor abdominal & 6 & 33.3 & 15 & 33.3 & 14 & 8.0 & 9 & 5.6 & 1.00 & $<0.01^{\dagger}$ \\
\hline
\end{tabular}

fue más común aquellos con FMMR (98.4\%) y las artralgias $(87.9 \%)$ en quienes padecieron chikungunya. El exantema que involucraba plantas y palmas fue observado en $88.7 \%$ de los pacientes con FMMR, pero solo en $7.8 \%$ de los sujetos con chikungunya y no fue observado en pacientes con dengue $(p<0.01)$. El edema que involucraba párpados (32.3\%), muñecas $(30.6 \%)$ o tobillos $(29.0 \%)$ fue más frecuente en el grupo con FMMR en comparación con el de pacientes con chikungunya (1.4, 2.1 y $5.0 \%)$, mientras que los pacientes con infección por dengue no presentaron edema $(p<0.01)$. El prurito ocurrió en pacientes con chikungunya (23.2 \%) y dengue (18.3 \%), pero estuvo ausente en el grupo con FMMR $(p<0.01)$. No se observaron otras diferencias (Tabla 1).

En relación con las características de laboratorio, los pacientes con FMMR tuvieron altos conteos iniciales de células blancas en sangre $(11.5 \pm 8.1 / \mu \mathrm{L})$ en comparación con los pacientes con dengue $(4.9 \pm 2.1 / \mu \mathrm{L})$ y chikungunya $(6.3 \pm 2.3 / \mu \mathrm{L})$, así como niveles más altos de bilirrubina total $(3.8 \pm 2.4 \mathrm{mg} / \mathrm{dL})$, principalmente por incremento en los niveles de bilirrubina directa; los pacientes con dengue $(0.5 \pm 0.4 \mathrm{mg} / \mathrm{dL})$ y chikungunya $(0.5 \pm 0.4 \mathrm{mg} / \mathrm{dL})$ mostraron niveles más bajos de bilirrubina total y la diferencia fue en gran medida debida a bilirrubina indirecta $(p<0.01)$.

Por otra parte, conteos más bajos de plaquetas $(30.2 \pm 26.910 / \mu \mathrm{L})$, niveles más bajos de sodio $(129.9 \pm 5.9 \mathrm{mEq} / \mathrm{L})$, de proteínas totales $(4.6 \pm 1.2 \mathrm{mg} / \mathrm{dL})$ y de albúmina $(2.5 \pm 0.7 \mathrm{~g} / \mathrm{dL})$ fueron observados en pacientes con FMMR, comparados con los individuos con dengue y chikungunya; las diferencias fueron estadísticamente significativas $(p<0.01)$. Niveles más altos de creatinina sérica se 
Tabla 2. Hallazgos clínicos y de laboratorio en el momento de la hospitalización en 399 pacientes con fiebre manchada de las Montañas Rocosas, dengue y chikungunya. Sonora, Mexico. 2004-2016

\begin{tabular}{|c|c|c|c|c|c|c|}
\hline Indicador & $\begin{array}{l}\text { FMMR }<5 \text { días } \\
\text { de evolución } \\
(n=18)\end{array}$ & $\begin{array}{l}\text { FMMR } \geq 5 \text { días } \\
\text { de evolución } \\
\quad(n=45)\end{array}$ & $\begin{array}{l}\text { Dengue } \\
(n=175)\end{array}$ & $\begin{array}{l}\text { Chikungunya } \\
\qquad(n=161)\end{array}$ & $p_{1}^{*}$ & $p_{2}^{\ddagger}$ \\
\hline Edad (años) & $23.3 \pm 20.0$ & $22.4 \pm 20.6$ & $34.0 \pm 20.2$ & $36.4 \pm 16.9$ & 0.78 & $<0.01^{\dagger}$ \\
\hline Estancia intrahospitalaria (días) & $6.7 \pm 8.9$ & $4.8 \pm 7.8$ & $4.6 \pm 2.9$ & $4.1 \pm 3.2$ & 0.05 & $<0.01^{\dagger}$ \\
\hline Tiempo de evolución (días) & $3.4 \pm 0.8$ & $6.3 \pm 2.3$ & $3.0 \pm 1.9$ & $4.4 \pm 2.9$ & $<0.01^{\dagger}$ & $<0.01^{\dagger}$ \\
\hline Hemoglobina, g/dL (ref. 11.5-18.0) & $13.8 \pm 4.9$ & $12.2 \pm 2.2$ & $14.0 \pm 6.2$ & $13.3 \pm 1.3$ & 0.36 & $<0.01^{\dagger}$ \\
\hline Hematocrito, \% (ref. 35.0-52.0) & $35.1 \pm 9.1$ & $35.8 \pm 6.4$ & $40.3 \pm 3.9$ & $40.0 \pm 3.8$ & 0.84 & $<0.01^{\dagger}$ \\
\hline 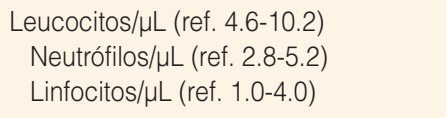 & $\begin{aligned} 9.6 & \pm 6.3 \\
11.2 & \pm 13.2 \\
1.4 & \pm 1.8\end{aligned}$ & $\begin{array}{c}12.3 \pm 8.7 \\
10.2 \pm 7.5 \\
1.1 \pm 1.3\end{array}$ & $\begin{array}{l}4.9 \pm 2.2 \\
3.2 \pm 2.1 \\
1.0 \pm 0.7\end{array}$ & $\begin{array}{l}6.3 \pm 2.3 \\
4.6 \pm 2.1 \\
1.1 \pm 0.9\end{array}$ & $\begin{array}{l}0.24 \\
0.57 \\
0.68\end{array}$ & $\begin{array}{c}<0.01^{\dagger} \\
<0.01^{\dagger} \\
0.04^{\dagger}\end{array}$ \\
\hline Plaquetas $10^{3} / \mu \mathrm{L}$ (ref. 150.0-450.0) & $41.9 \pm 30.7$ & $25.2 \pm 23.7$ & $166.4 \pm 156.8$ & $226.0 \pm 78.9$ & 0.05 & $<0.01^{\dagger}$ \\
\hline $\begin{array}{l}\text { Tiempo de protrombina, segundos } \\
\text { (ref. 11.1-14.1) }\end{array}$ & $18.6 \pm 3.3$ & $19.1 \pm 9.5$ & $14.3 \pm 2.5$ & $14.1 \pm 2.0$ & 0.18 & $<0.01^{\dagger}$ \\
\hline $\begin{array}{l}\text { Tiempo de tromboplastina parcial, } \\
\text { segundos (ref. 20.0-40.0) }\end{array}$ & $63.4 \pm 33.5$ & $57.5 \pm 37.6$ & $37.4 \pm 11.0$ & $33.9 \pm 4.4$ & 0.44 & $<0.01^{\dagger}$ \\
\hline Bilirrubina total, mg/dL (ref. 0.0-1.1) & $2.9 \pm 2.3$ & $4.0 \pm 2.5$ & $0.5 \pm 0.4$ & $0.5 \pm 0.4$ & 0.29 & $<0.01^{\dagger}$ \\
\hline Bilirrubina directa, mg/dL (0.0-0.25) & $2.7 \pm 2.0$ & $3.1 \pm 2.0$ & $0.2 \pm 0.2$ & $0.2 \pm 0.1$ & 0.65 & $<0.01^{\dagger}$ \\
\hline Bilirrubina indirecta, mg/dL (0.0-0.85) & $0.4 \pm 0.3$ & $1.0 \pm 0.8$ & $0.3 \pm 0.2$ & $0.3 \pm 0.3$ & $0.04^{+}$ & $<0.01^{\dagger}$ \\
\hline $\begin{array}{l}\text { Transaminasa glutámico oxalacética, } \\
\text { UI/L (ref. 0.0-32.0) }\end{array}$ & $221.6 \pm 131.6$ & $283.5 \pm 168.6$ & $82.9 \pm 194.1$ & $31.1 \pm 16.2$ & 0.22 & $<0.01^{\dagger}$ \\
\hline $\begin{array}{l}\text { Transaminasa glutámico pirúvica, UI/L } \\
\text { (ref. 7.0-31.0) }\end{array}$ & $85.6 \pm 52.4$ & $99.8 \pm 51.8$ & $59.0 \pm 92.6$ & $41.9 \pm 64.8$ & 0.33 & $<0.01^{\dagger}$ \\
\hline $\begin{array}{l}\text { Lactato deshidrogenasa, UI/L (ref. } \\
\text { 240.0-400.0) }\end{array}$ & $1343.9 \pm 835.5$ & $1253.0 \pm 652.6$ & $550.2 \pm 430.3$ & $244.7 \pm 89.6$ & 0.53 & $<0.01^{\dagger}$ \\
\hline Creatinina sérica, mg/dL (ref. 0.5-0.9) & $1.9 \pm 1.7$ & $1.9 \pm 1.4$ & $0.7 \pm 0.4$ & $0.8 \pm 0.3$ & 0.61 & $<0.01^{\dagger}$ \\
\hline Urea, mg/dL (ref. 16.6-48.5) & $64.0 \pm 43.8$ & $93.9 \pm 54.6$ & $18.9 \pm 10.1$ & $24.6 \pm 16.2$ & 0.08 & $<0.01^{\dagger}$ \\
\hline $\begin{array}{l}\text { Nivel de sodio sanguíneo, mEq/L (ref. } \\
\text { 136.0-146.0) }\end{array}$ & $125.4 \pm 34.3$ & $128.3 \pm 5.9$ & $136.5 \pm 3.1$ & $137.3 \pm 3.7$ & $<0.01^{\dagger}$ & $<0.01^{\dagger}$ \\
\hline $\begin{array}{l}\text { Nivel de potasio sanguíneo, mEq/L } \\
\text { (ref. 3.5-5.1) }\end{array}$ & $4.2 \pm 0.1$ & $3.5 \pm 0.4$ & $4.0 \pm 0.6$ & $3.9 \pm 0.4$ & $0.04^{+}$ & $0.03^{+}$ \\
\hline $\begin{array}{l}\text { Nivel de cloro sanguíneo, mEq/L (ref. } \\
\text { 97.0-111.0) }\end{array}$ & $93.0 \pm 5.0$ & $91.4 \pm 6.7$ & $98.8 \pm 4.0$ & $101.2 \pm 4.2$ & $0.36^{* *}$ & $<0.01^{\dagger}$ \\
\hline $\begin{array}{l}\text { Proteínas séricas totales, mg/dL (ref. } \\
6.4-8.3 \text { ) }\end{array}$ & $4.4 \pm 1.4$ & $4.7 \pm 1.1$ & $6.6 \pm 0.8$ & $6.1 \pm 0.7$ & $0.37^{\star \star}$ & $<0.01^{+}$ \\
\hline Albumina sérica, g/dL (ref. 3.8-5.4) & $2.3 \pm 0.9$ & $2.6 \pm 0.6$ & $3.9 \pm 0.6$ & $3.7 \pm 0.7$ & $0.13^{\star \star}$ & $<0.01^{\dagger}$ \\
\hline Globulina, g/dL (ref. 2.1-3.8) & $2.0 \pm 0.6$ & $2.1 \pm 0.7$ & $2.8 \pm 0.6$ & $2.5 \pm 0.3$ & $0.6^{\star *}$ & $<0.01^{\dagger}$ \\
\hline
\end{tabular}

*Basado en una prueba de $U$ de Mann-Whitney. **Basado en una prueba de t de Student. 'Estadísticamente significativo. *Basado en una prueba de ANOVA de una vía. $p_{1}$ Comparación entre FMMR $<5$ días $y \geq 5$ días. $p_{2}$ Comparación entre los cuatro grupos. $\mathrm{FMMR}$ = fiebre manchada de las Montañas Rocosas.

registraron en pacientes con FMMR $(1.9 \pm 1.5 \mathrm{mg} / \mathrm{dL})$, comparados con los infectados por los virus del dengue $(0.7 \pm 0.4 \mathrm{mg} / \mathrm{dL})$ y de chikungunya $(0.8 \pm 0.3 \mathrm{mg} / \mathrm{dL})$, $\mathrm{p}<0.01$ (Tabla 2).

\section{Discusión}

Este estudio compara y contrasta varios patrones clínicos y de laboratorio de pacientes diagnosticados 
con FMMR, dengue y chikungunya en una región de México donde estas tres enfermedades son endémicas. El reconocimiento de las diferencias puede guiar a los médicos para establecer un diagnóstico oportuno en regiones donde estas enfermedades son endémicas e iniciar apropiadamente terapia antibiótica para reducir el riesgo de desenlaces fatales en los pacientes con FMMR. La sospecha clínica es crítica debido a la conocida gravedad de una proporción de pacientes infectados por Rickettsia rickettsii en el noroeste de México, situación agravada por los síntomas inespecíficos en las etapas tempranas de esta enfermedad. ${ }^{8,9}$

En nuestra serie, tres características clínicas primarias distinguen estas enfermedades. El exantema petequial se presentó en todos los grupos (Figura 1), aunque se observaron diferencias en su prevalencia y distribución con una ocurrencia notable $(88.7 \%)$ de petequias en palmas y plantas en el grupo con FMMR (Tabla 1). Las petequias son un signo distintivo de daño vascular grave en estadios tardíos de la enfermedad..$^{10}$ El segundo signo distintivo es el edema en extremidades y párpados, el cual puede ayudar a sospechar FMMR en etapas tardías. Ambas características son explicadas por una disfunción endotelial diseminada y replicación bacteriana, así como por la estimulación del estrés oxidativo, que resulta en daño a las células endoteliales. ${ }^{11}$ Además, el prurito y el exantema maculopapular han sido descritos previamente en pacientes con dengue y chikungunya, ${ }^{12,13} \sin$ embargo, esos síntomas no han sido documentados en pacientes con FMMR, lo cual fue también observado en nuestra serie; por lo tanto, su presencia debe guiar a los médicos a sospechar dengue y chikungunya. Por otra parte, el exantema y el edema son características típicas de un estadio avanzado de FMMR y puede ser solo útil para guiar decisiones medicas en esta situación, pero no debe ser utilizados para la sospecha clínica temprana de las enfermedades en el primer contacto médico.

La mayoría de los pacientes con FMMR mostraron conteos bajos de plaquetas, así como tiempos de protrombina y tromboplastina más prolongados que los de los pacientes con dengue y chikungunya $(p<0.01)$ (Tabla 2). Por otra parte, el involucramiento hepático ha sido documentado en pacientes con FMMR, el cual se caracteriza por impedimento en la excreción de bilirrubina conjugada y elevación de transaminasas séricas, secundarios tanto a muerte focal de hepatocitos y colestasis ductal debido a grave inflamación de las tríadas portales asociada a edema,${ }^{14}$ con elevación de la bilirrubina directa. Esto es diferente de lo

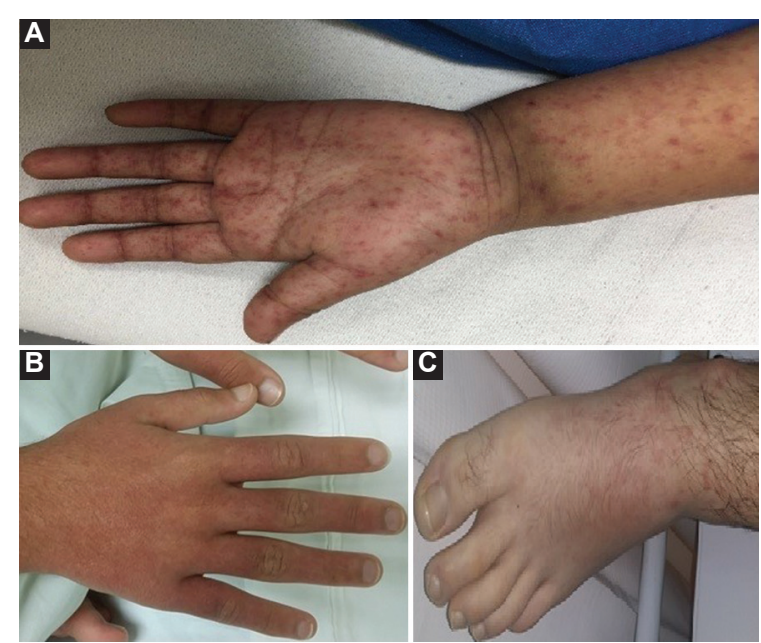

Figura 1. Características del exantema en pacientes con FMMR, dengue y chikungunya. Sonora, México. A) Paciente con FMMR con exantema petequial que involucraba palma y edema de muñeca. B) Paciente con dengue con exantema petequial en manos, pero no en palmas. C) Paciente con chikungunya con exantema petequial en pie, pero no en planta.

observado en dengue y chikungunya $(p<0.05)$ (Tabla 2), en los cuales se atribuye a incremento de la bilirrubina directa a expensas de la indirecta, secundario a destrucción periférica de eritrocitos. ${ }^{12}$

Como ha sido mencionado, el mecanismo patológico de las enfermedades originadas por rickettsias provoca daño endotelial con incremento subsecuente en la permeabilidad vascular, ${ }^{11}$ mecanismo que puede estar asociado al menor conteo de niveles de sodio sérico y de proteínas totales séricas, que se presenta predominantemente en los pacientes con FMMR comparados con aquellos con dengue y chikungunya $(p<0.01)$ (Tabla 2). Del mismo modo, el daño endotelial con permeabilidad incrementada genera disminución en el volumen intravascular, que deriva en un estado de hipovolemia, el cual, aunque en el inicio de la enfermedad pueda llevar a falla prerrenal, si persiste puede generar un necrosis tubular aguda; ambos fenómenos pueden llevar a azotemia. ${ }^{15}$

Del mismo modo, aunque las muertes por dengue hayan sido reportadas en años previos, con la introducción generalizada de las pruebas por PCR para Rickettsia rickettsii en la región, una disminución en los reportes de muertes por dengue han sido observadas, siendo de 22 en 2014, cinco en 2015 y solo uno en el año 2016, ${ }^{16}$ mientras que un incremento en muertes por FMMR ha sido notado en el mismo periodo, lo cual podría ser explicado por limitaciones en la capacidad diagnóstica a nivel regional. 
Nuestro estudio tiene limitaciones, una es la incapacidad para identificar coinfecciones de dengue, chikungunya y FMMR, debido a que el algoritmo actual se detiene cuando se obtiene un resultado positivo de cualquiera de las pruebas diagnósticas. Adicionalmente, hubo un potencial sesgo de selección debido a que en esta evaluación solo fueron incluidos pacientes hospitalizados por FMMR, mientras que la mayoría de los pacientes con infección por virus de dengue y chikungunya fueron tratados como pacientes externos.

\section{Conclusión}

En regiones donde se presentan en forma simultánea FMMR, dengue y chikungunya, la diferenciación clínica entre estas enfermedades es un reto y una prioridad para los médicos de primer contacto. Esto puede aplicar en múltiples regiones de Latinoamérica, incluidos países como Panamá, Brasil, Costa Rica, Argentina y Colombia. ${ }^{17-21}$ Nuestros hallazgos sugieren que algunos signos que puede ser identificados en una exploración física cuidadosa como exantema, edema y prurito, en adición al patrón de datos de laboratorio como bilirrubina o conteo de plaquetas, pueden ser indicadores útiles para distinguir a los pacientes en estadios avanzados de FMMR de aquellos con dengue y chikungunya.

\section{Agradecimientos}

Estamos agradecidos con el doctor Christopher Paddock por los valiosos comentarios realizados al contenido de este manuscrito.

\section{Conflicto de intereses}

Los autores declaran no tener conflicto de intereses alguno.

\section{Financiamiento}

Los autores no recibieron patrocinio para llevar a cabo este artículo.

\section{Responsabilidades éticas}

Protección de personas y animales. Los autores declaran que para esta investigación no realizaron experimentos en seres humanos ni en animales.
Confidencialidad de los datos. Los autores declaran que siguieron los protocolos de su centro de trabajo sobre la publicación de datos de pacientes.

Derecho a la privacidad y consentimiento informado. Los autores obtuvieron el consentimiento informado de los pacientes o sujetos referidos en el artículo. Este documento obra en poder del autor de correspondencia.

\section{Bibliografía}

1. Biggs HM, Behravesh CB, Bradley KK, Dahlgren FS, Drexler NA, Dumler S, et al. Diagnosis and management of tickbornerickettsial diseases: Rocky Mountain spotted fever and other spotted fever group rickettsioses, ehrlichioses, and anaplasmosis - United States. MMWR Recomm Rep. 2016;65:1-44.

2. Ricketts HT. Some aspects of Rocky Mountain spotted fever as shown by recent investigations. 1909. Rev Infect Dis. 1991;13:1227-1240.

3. Bustamante M. A new rickettsiosis in Mexico. Existence of the American spotted fever in the States of Sinaloa and Sonora. Rev Inst Sal Enf Trop. 1943:4:189-211

4. Pan American Health Organization. Dengue: guidelines for patient care in the Region of the Americas. EE. UU.: Pan American Health Organization; 2016.

5. Pan American Health Organization. Preparedness and response for Chikungunya virus: introduction in the Americas. EE. UU.: United States, National Center for Emerging and Zoonotic Infectious Diseases/Pan American Health Organization; 2011

6. Labruna MB, Whitworth T, Horta MC, Bouyer DH, McBride JW, Pinter A, et al. Rickettsia species infecting Amblyommacooperi ticks from an area in the state of Sao Paulo, Brazil, where Brazilian spotted fever is endemic. J ClinMicrobiol. 2004;42:90-98.

7. Kato CY, Chung IH, Robinson LK, Austin AL, Dasch GA, Massung RF. Assessment of real time PCR assay for detection of Rickettsia spp. and Rickettsia rickettsii in banked clinical samples. J ClinMicrobiol. 2013;52:314-317.

8. Álvarez-Hernández G, Murillo-Benítez C, Candia-Plata MC, Moro M. Clinical profile and predictors of fatal Rocky Mountain spotted fever in children from Sonora, Mexico. Pediatr Infect Dis J. 2015;34:125-130.

9. Delgado-de la Mora J, Licona-Enríquez JD, Leyva-Gastélum M, Delgado-de la Mora D, Rascón-Alcantar A, Álvarez-Hernández G. Una serie fatal de casos de fiebre manchada de las montañas rocosas en Sonora, México. Biomedica. 2018;38:69-76.

10. Dumler JS. Intracellular pathogens II. Rickettsiales. En: Palmer GH, Azad AF (editores). Clinical disease: current treatment and new challenges. EE. UU.: American Society for Microbiology Press; 2012.

11. Walker DH, Ismail N. Emerging and re-emerging rickettsioses: endothelial cell infection and early disease events. Nat Rev Microbiol. 2008:6:375-386.

12. Simmons CP, Farrar JJ, Chau NV, Wills B. Dengue. N Engl J Med. 2012;366:1423-1432.

13. Ratsitorahina M, Harisoa J, Ratovonjato J, Biacabe S, Reynes JM, Zeller $\mathrm{H}$, et al. Outbreak of dengue and Chikungunya fevers, Toamasina, Madagascar, 2006. Emerg Infect Dis. 2008;14:1135-1137.

14. Adams JS, Walker DH. The liver in Rocky Mountain spotted fever. Am J ClinPathol. 1981;75:156-161.

15. Walker DH, Mattern WD. Acute renal failure in Rocky Mountain spotted fever. Arch Intern Med. 1979;139:443-448.

16. Secretaría de Salud [Internet]. México: Panorama epidemiológico de dengue 2016; 2017.

17. Tribaldos M, Zaldívar $Y$, Bermúdez S, Samudio F, Mendoza $Y$, Martínez AA, et al. Rocky Mountain spotted fever in Panama: a cluster description. J Infect Dev Ctries. 2011;5:737-741.

18. Szabó MP, Pinter A, Labruna MB. Ecology, biology and distribution of spotted-fever tick vectors in Brazil. Front Cell Infect Microbiol. 2013;3:27.

19. Argüello AP, Hun L, Rivera $P$, Taylor L. Case report: a fatal urban case of Rocky Mountain spotted fever presenting an eschar in San Jose, Costa Rica. Am J Trop Med Hyg. 2012;2:345-348.

20. Paddock CP, Fernández S, Echenique GA, Sumner JW, Reeves WK, Zaki WR, et al. Rocky Mountain spotted fever in Argentina. Am J Trop Med Hyg. 2008;4:687-692.

21. Hidalgo M, Orejuela L, Fuya $P$, Carrillo $P$, Hernández J, Parra E, et al. Rocky Mountain spotted fever, Colombia. Emerg Infect Dis. 2007;7: 1058-1060. 